\title{
VHF RADIO INTERFEROMETRY OF LIGHTNING
}

\author{
P.R. KREHBIEL, X.M. SHAO, R.J. THOMAS
}

New Mexico Institute of Mining and Technology, Socorro, NM 87801

C.T. RHODES

Los Alamos National Laboratories, Los Alamos, NM 87545

C.O. HAYENGA

Array Technology Corp., Boulder, CO 80301

\begin{abstract}
Lightning discharges radiate strongly at radio frequencies, and studies of the radiation provide valuable information on lightning breakdown processes. Interferometric techniques can be used to locate the numerous radiation events as a function of time during a discharge and to generate images of the developing lightning channels inside a storm, where they are obscured from view at optical wavelengths.
\end{abstract}

Interferometric techniques have and are being successfully applied to the study of radio frequency radiation from lightning discharges by groups both in the U.S. (Hayenga and Warwick, 1981; Rhodes, 1989) and in France (Richard and Auffray, 1985). The lightning interferometers have time resolutions down to one microsecond and utilize relatively short baselines to locate the radiation centroid as a function of time during a discharge. The radiation is assumed to be localized at each instant of time and the direction of its centroid is determined from the phase differences of the received signal at various pairs of antennas, using standard interferometric analyses. The results augment and improve upon those obtained by time-of-arrival techniques (e.g. Proctor, 1988), which locate the sources of impulsive radiation events produced by lightning.

It was originally not known if lightning radiation was sufficiently localized to be studied using simple interferometric techniques. The expectation that lightning would radiate simultaneously at numerous different locations caused the interferometric technique to be controversial, but observational results have shown that the radiation is indeed relatively well-localized much of the time during a lightning event. The dominant radiation at VHF and UHF appears to occur at the leading edge of negative breakdown streamers, i.e. streamers which progress by accelerating electrons away from their advancing tips. Positive streamers or the positive end of negative breakdown events do not appear to radiate strongly. In addition, little $\mathrm{RF}$ radiation is produced by charge flow along already-conducting channels.

Figure 1 shows the antenna configuration for the lightning interferometer that has been developed at New Mexico Tech. Five vertical monopole antennas are located on a horizontal ground plane to form short $(1 / 2 \lambda)$ and long $(4 \lambda)$ baselines in each of two orthogonal directions. The short baselines provide 
coarse but unambiguous estimates of the source direction that are used to resolve the ambiguities of the long baseline measurements. The interferometer operates at a center frequency of $274 \mathrm{MHz}$ with a bandwidth of $6 \mathrm{MHz}$ and a time resolution of $1 \mu \mathrm{s}$. The center frequency was chosen to give good signal strength, which decreases with increasing frequency, while keeping the size of the array relatively small (and the consequent signal decorrelation across the array). Constant-phase limiting amplifiers were used in the receivers to accomodate the large dynamic range of the RF radiation signals.

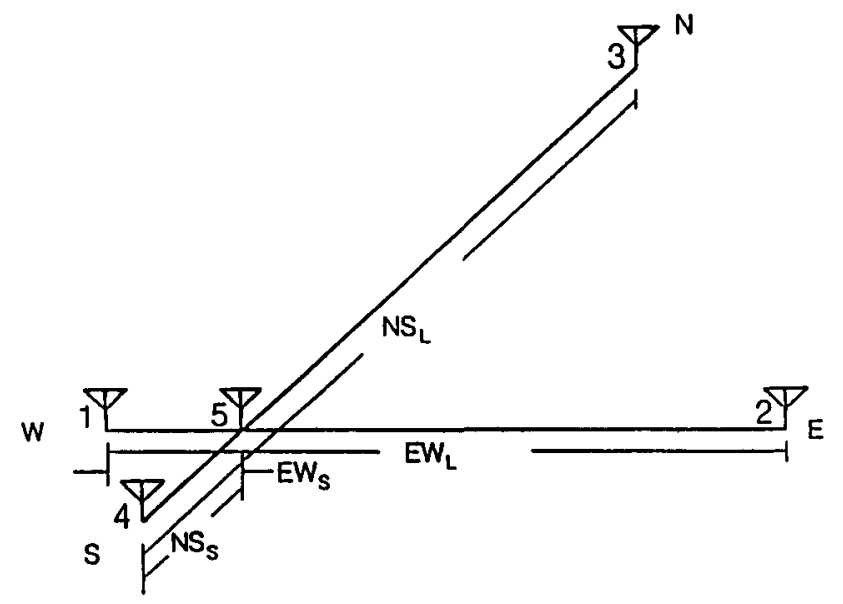

Fig. 1. Antenna configuration of lightning interferometer.

Figure 2a shows short baseline phase values for a 'leader' event which initiated a stroke between the cloud and ground. The normalized phase differences from the orthogonal baselines are plotted in $x$-y format and exhibit substantial variability in their values. The square grid indicates the fringes of the long baselines, whose phase differences give a fine estimate of the source location within a fringe. Figure $2 \mathrm{~b}$ shows the result of initially combining the short and long baseline data. Substantial aliasing is seen in the phase values that is not real but results from the variability of the short baseline data. The leader radiation was continuous in space and time, as can be seen when the aliasing is removed (Figure 2c).

If one considers the radiation sources to originate on a celestial sphere of unit radius, the phase plane results give the projection of the sources onto the plane of the antenna array. The inscribed (unit) circle corresponds to the horizon and the center of the phase plane corresponds to the zenith. In time sequence, the radiation sources for the leader began in the southeast and progressed overhead and to the horizon (ground) north of the interferometer. The azimuth-elevation plot of Figure $2 \mathrm{~d}$ graphically reveals the channel to ground and indicates a horizontal orientation of the in-cloud part of the channel. Figure 2e shows various time waveforms for the leader, including the logarithmic radiated power and atmospheric electric field change. The leader lasted about $2 \mathrm{~ms}$ and progressed at speeds between about $10^{6}$ and $10^{7} \mathrm{~m} / \mathrm{s}$. 

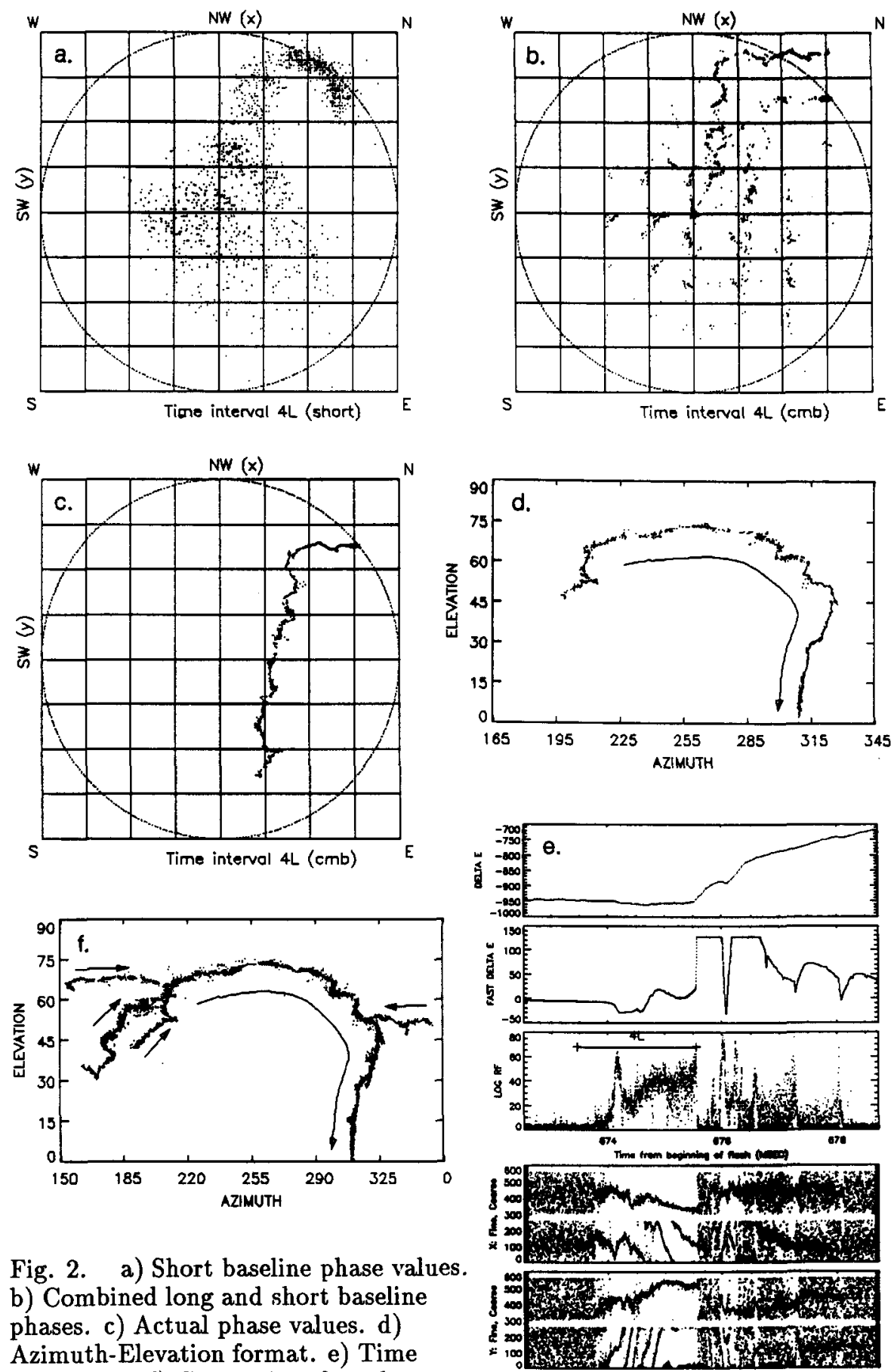

Fig. 2. a) Short baseline phase values. b) Combined long and short baseline phases. c) Actual phase values. d) Azimuth-Elevation format. e) Time waveforms. f) Composite of results. 
Because the phase values correspond to projected source locations, sources at low elevation angles are compressed toward the horizon circle in the phase plane and their elevation angles are not well-determined by the interferometer. The interferometer is thus 'myopic', i.e. it tends to work best for lightning that is overhead (or, if the array were pointable, for lightning normal to the plane of the antenna array). The greatest difficulty in using the interferometer to study lightning radiation, however, stems from the need to correct the substantial aliasing and systematic errors in the source locations illustrated in Figure 2b. The causes of these problems are systematic and random errors in the short baseline phases. The systematic errors arise from a combination of effects, including antenna interaction and a non-ideal ground plane, and can be seen by comparing the source locations in Figure $2 \mathrm{~b}$ with the actual locations of Figure 2c. Because the systematic errors are a constant function of the array configuration, they can be estimated from the data of well-located events (as above) and used to correct data for other events. This procedure is limited somewhat by the random errors of the phase measurements but has been found to work fairly well in resolving the fringe ambiguities.

Figure 2f shows a composite of selected results for the entire lightning flash. The flash lasted 1.2 seconds and produced 5 strokes along the channel to ground, each initiated by successively longer leaders along the main channel and different branches. Additional activity prior to, in between, and after the strokes repeatedly retraced and extended the channels, invariably as negative streamers which propagated in the directions indicated. Each stroke lowered negative charge to ground. The elevation angles of the left-hand channels decreased as they became more distant from the interferometer. The channel to ground emerged from cloud base at about $25^{\circ}$ elevation; all of the channels above this point were inside the cloud and would have been obscured optically. Two stub-like branches are seen in the downward part of the channel to ground.

A number of improvements are possible for the interferometer, which is still under development. These include the use of increased numbers of baselines or baselines of non-integer ratios to reduce the effects of systematic errors, and the use of different types of antennas having reduced interaction and more omnidirectional responses. Three-dimensional and real-time systems are also possible (Richard et al., 1988). Valuable scientific results continue to be obtained as development of the interferometric techniques proceeds, however.

\section{REFERENCES}

Hayenga, C. and J. Warwick, 1981, J. Geophys. Res., 86, 7451.

Proctor, D. 1988, J. Geophys. Res., 93, 12683.

Rhodes, C. 1989, Ph.D. Dissertation, N.M. Inst. Min. and Tech, Socorro, NM. Richard, P. and G. Auffray, 1985, Radio Sci., 20, 171.

Richard, P., A. Soulage, P. LaRoche, and J. Appel, 1988, in Proceedings Intn'l. Aerospace and Ground Conf. Lightning and Static Electricity, NOAA, U.S. Dept. Comm., p. 383. 